February 2017

\title{
Coinfection with Lichtheimia corymbifera and Aspergillus flavus in an Immune-Competent Patient Mimicking as Pulmonary-Renal Syndrome
}

\author{
Ali Bin Sarwar Zubairi \\ Aga Khan University, ali.zubairi@aku.edu \\ Farah Idress \\ Aga Khan University, farah.idrees@aku.edu \\ Kauser Jabeen \\ Agha Khan University, kausar.jabeen@aku.edu \\ Saima Kamal \\ Aga Khan University, saima.kamal@aku.edu \\ Afia Zafar \\ Agha Khan University, afia.zafar@aku.edu
}

Follow this and additional works at: https://ecommons.aku.edu/pakistan_fhs_mc_med_med

Part of the Pathology Commons, and the Pulmonology Commons

\section{Recommended Citation}

Zubairi, A., Idress, F., Jabeen, K., Kamal, S., Zafar, A. (2017). Coinfection with Lichtheimia corymbifera and Aspergillus flavus in an Immune-Competent Patient Mimicking as Pulmonary-Renal Syndrome. Mycopathologia, 182(7-8), 727-731.

Available at: https://ecommons.aku.edu/pakistan_fhs_mc_med_med/288 


\title{
Coinfection with Lichtheimia corymbifera and Aspergillus flavus in an Immune-Competent Patient Mimicking as Pulmonary-Renal Syndrome
}

\author{
Ali Bin Sarwar Zubairi · Farah Idrees · Kauser Jabeen · Saima Kamal • Afia Zafar
}

Received: 26 November 2015/ Accepted: 26 January 2017/Published online: 14 February 2017

(C) Springer Science+Business Media Dordrecht 2017

\begin{abstract}
Lichtheimia corymbifera and Aspergillus flavus pulmonary coinfection has been rarely reported in immune-competent patients. We report case of a young male who presented with clinical features of pulmonary-renal syndrome and was later diagnosed to have bilateral polymicrobial fungal lung infection.
\end{abstract}

Keywords Lichtheimia corymbifera $\cdot$ Aspergillus flavus $\cdot$ Coinfection $\cdot$ Invasive mycosis

\section{Introduction}

Invasive fungal infections have remained a major cause of morbidity and mortality in immunocompromised individuals, particularly those with hematological malignancies, solid organ transplant and neutropenia [1]. Polymicrobial pulmonary fungal infection is a rare entity reported mainly in severely immunodeficient patients [2-7]. Aspergillus species and mucoraceous molds have been implicated mainly as a cause of coinfection. Underlying risk factors in

A. B. S. Zubairi $(\bowtie) \cdot$ F. Idrees · S. Kamal

Department of Medicine, Aga Khan University Hospital, Karachi, Pakistan

e-mail: ali.zubairi@aku.edu

K. Jabeen $\cdot$ A. Zafar

Department of Pathology and Laboratory Medicine, Aga

Khan University Hospital, Karachi, Pakistan these cases have varied from poorly controlled diabetes, chronic steroid use, leukemia, HIV and cardiac transplant [2-7]. Agents of mycosis reported in dual infections have been Aspergillus fumigatus, Aspergillus flavus, Aspergillus alliaceus, Mucor species, Lichtheimia corymbifera, mucoraceous mold (not speciated). Patient outcome with fungal coinfection has been poor with high mortality rates [2-7].

\section{Case Report}

A 45-year-old male with no prior comorbid presented to the Emergency department with history of productive cough, high grade fever and dyspnea for a week and submassive hemoptysis for past one day. There was no history of smoking, and he denied history of other substance abuse. His past, family and drug history was not significant. He had no history of any previous infections or surgical intervention. On physical examination, he was awake and alert, had a pulse of 118 beats per minute, respiratory rate of 32 breaths per minute and blood pressure of $140 / 90 \mathrm{mmHg}$ and was afebrile at the time of examination in emergency room. Blood oxygen saturation was $91 \%$ on 51 of oxygen. On chest auscultation, there were bilateral coarse crackles till mid lung zones. Cardiovascular, abdominal and neurological examination was normal. Laboratory data revealed creatinine of $3.5 \mathrm{mg} / \mathrm{dl}$, blood urea of $96 \mathrm{mg} / \mathrm{dl}$ and WBC count of $23 \times 10^{9} / 1$. His blood sugars were normal. Chest 
radiograph showed bilateral alveolar infiltrates which were denser in lower lung zones (Fig. 1). Blood and sputum cultures were obtained, and he was treated as severe community acquired pneumonia with imipenem, vancomycin and levofloxacin.

The chest X-ray and hypoxemia worsened within $24 \mathrm{~h}$ of admission requiring intubation and transfer to the intensive care unit. High-resolution CT of the chest and abdomen without contrast showed multiple patchy areas of consolidation and ground glass opacities in both lungs with underlying cystic and cavitary changes without mediastinal or hilar lymphadenopathy or pleural effusion (Fig. 2), while the abdominal examination was reported as normal. Intravenous contrast was not given due to deranged renal parameters. Bronchoscopy revealed inflamed bronchial mucosa and blood in the right bronchial tree. Multiple urinalyses showed microscopic hematuria and no red blood cell casts. A clinical diagnosis of pulmonaryrenal syndrome was made due to rapid deterioration of both respiratory and renal functions. Sequential bronchial lavage was not done due to high oxygen requirement. Nephrology and Infectious Disease consult was obtained, and they agreed with the diagnosis of pulmonary-renal syndrome. He was commenced on intravenous pulse steroids along with plasmapheresis.

Meanwhile microscopic examination of bronchoalveolar lavage (BAL) in mycology laboratory and cytopathology revealed presence of septate hyphae. Aseptate hyphae were not seen on initial microscopic examination. Gram, acid fast and

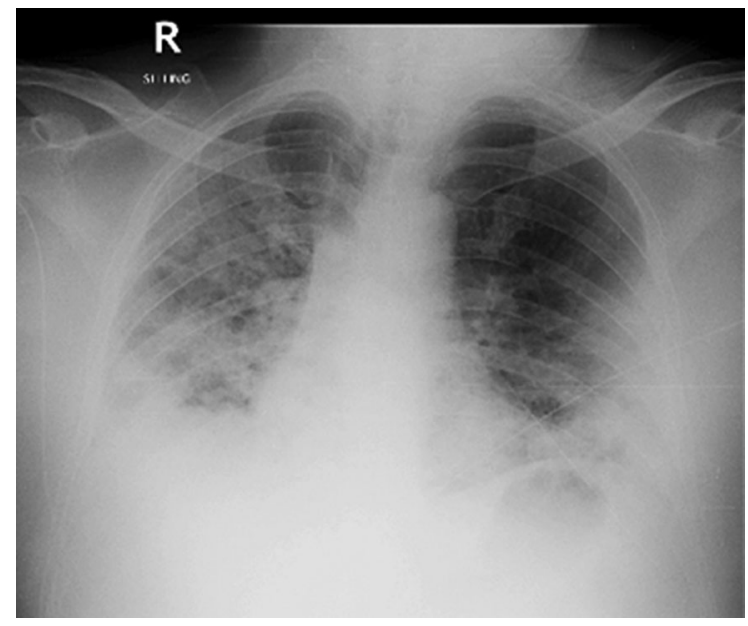

Fig. 1 Chest X-ray on admission showing bilateral alveolar lung infiltrates that are more dense in lower lung zones

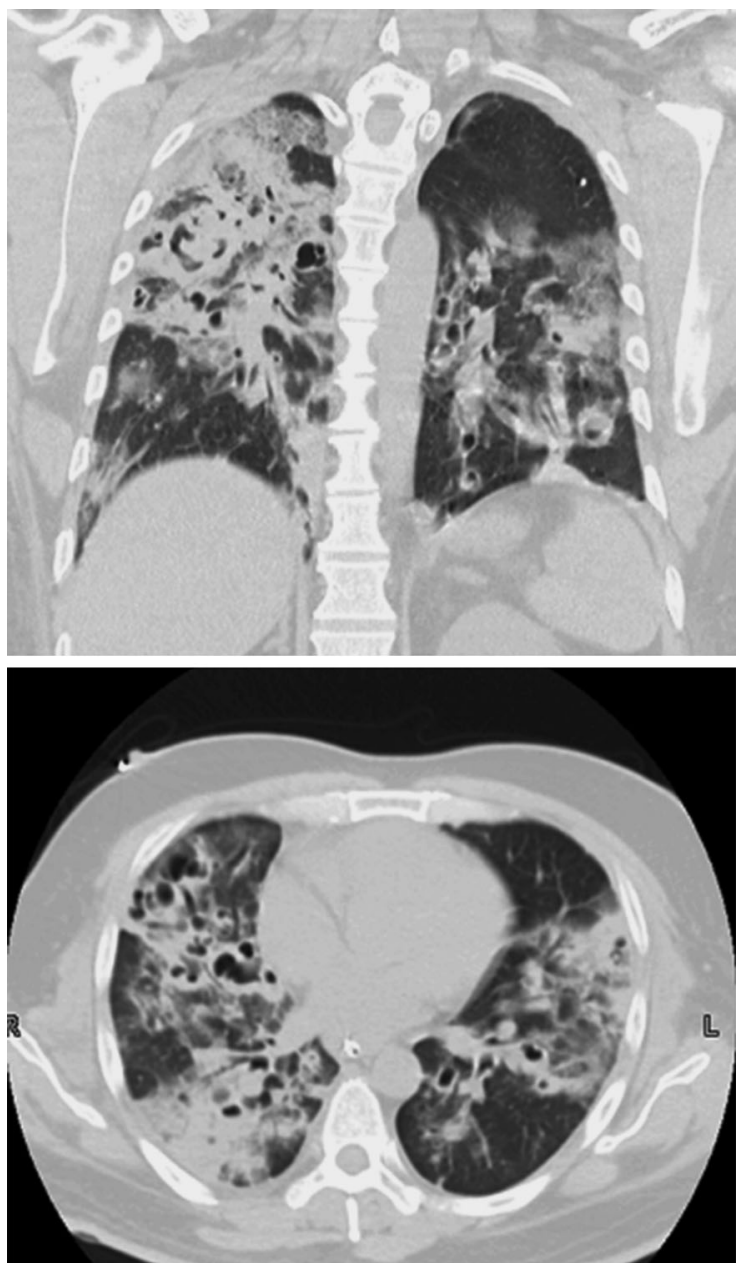

Fig. 2 Coronal and axial views of high-resolution CT of the chest (HRCT) show multiple patchy areas of consolidation and ground glass opacities in both lungs with underlying cystic and cavitary changes

immunofluorescent stain for Pneumocystis jirovecii were negative. Based on microscopic findings, the patient was started on amphotericin B. Bacterial and fungal cultures were set up in the laboratory; for fungi, Sabouraud dextrose agar plates were incubated at 25 and $37{ }^{\circ} \mathrm{C}$, potato agar and Mycosel agar were also used for fungal growth. For bacterial growth, chocolate, sheep blood and MacConkey's agars were used and incubated at $36{ }^{\circ} \mathrm{C}$ for $48 \mathrm{~h}$. There was growth of two types of molds on all non-selective plates and no bacterial growth $48 \mathrm{~h}$ later. These two molds were phenotypically identified $72 \mathrm{~h}$ later as $L$. corymbifera and A. flavus. At this point, voriconazole was added to the regimen, and steroids and 
plasmapheresis were stopped. Laboratory results of ANA, anti-dsDNA, c-ANCA, p-ANCA, (done by ELISA, DiaSorin, Italy), HIV, Hepatitis B and C virus were reported as negative. Bacterial and mycobacterial cultures in BAL were finally reported as negative. Blood cultures; the first set drawn before the administration of antibiotics and urine cultures were also reported as negative for any bacterial or fungal pathogens. The antibacterial drugs were stopped on day seven.

Patient eventually improved and was extubated after two days of ICU stay. His creatinine levels were normal $(1 \mathrm{mg} / \mathrm{dl})$ when he was shifted out of the ICU. The steroids were gradually tapered off. He was sent home in a stable clinical state without supplemental oxygen. The lung infiltrates on chest radiograph had completely resolved and renal function improved on a clinic follow-up after 2 weeks of hospital discharge (Fig. 3).

\section{Microbiological Diagnosis}

Fungal and bacterial cultures were performed using standard methodology [8]. After $24 \mathrm{~h}$ of incubation culture plates inoculated with BAL revealed abundant growth of whitish floccose colonies typical of a mucoraceous with a colorless reverse. Growth of a second type of mold with flat, granular, greenish colonies was also noted in Sabouraud dextrose agar

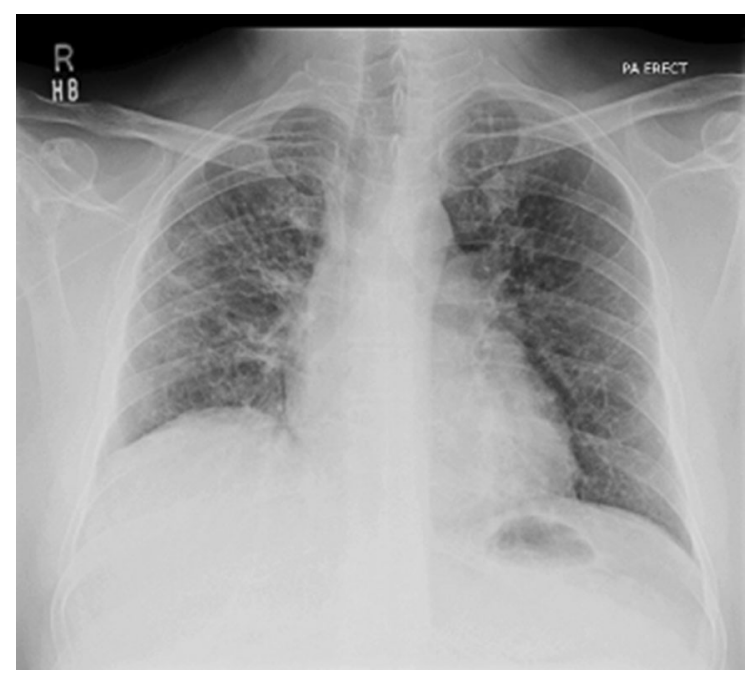

Fig. 3 Chest X-ray after 3 weeks of antifungal treatment showing complete resolution of lung infiltrates
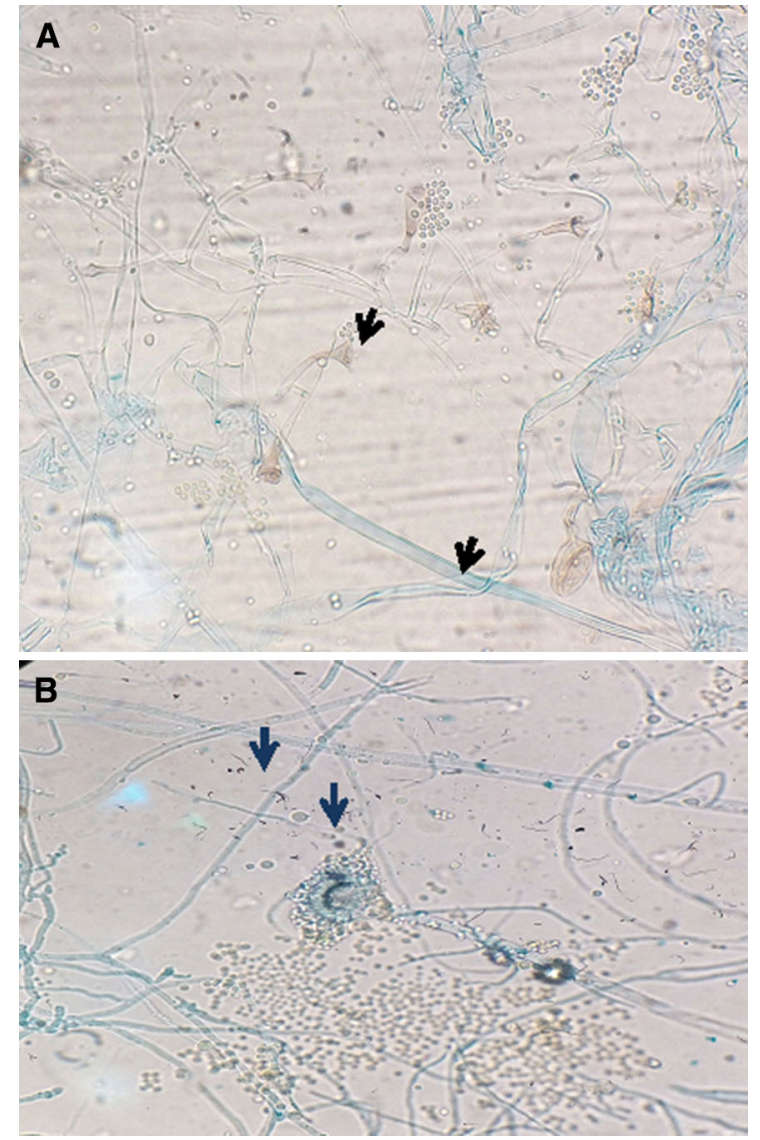

Fig. 4 Microscopic morphology of Lichtheimia corymbifera (a) and Aspergillus flavus (b). a Broad ribbon-like aseptate hyphae and sporangium of Lichtheimia corymbifera. b Thin, septate hyphae and head of Aspergillus flavus (arrow)

(both plates incubated at 25 and $37^{\circ} \mathrm{C}$ ), potato agar (to induce sporulation), blood and chocolate agar plates. Identification was made on the basis of gross appearance of colonies on plates and microscopic examination. Aspergillus flavus was identified by the presence of typical rough conidiophore, uniseriate and biseriate phialides covering entire vesicles (Fig. 4a) [9]. Lichtheimia corymbifera was identified on the basis of typical colony morphology, branching sporangiophore bearing small sporangia with funnel-shaped bases and a conical columella (Fig. 4b) [9].

\section{Discussion}

Invasive pulmonary mycosis has been rarely reported in patients with no apparent risk factors [10-16]. In 
most instances, extensive environmental exposure to fungal spores during occupational or recreational activities has been identified as an initiating event. Mixed pulmonary mycosis due to A. fumigatus and Rhizopus species in a previously healthy patient has reported in one patient with after near drowning [17]. This patient had a history of aspiration of muddy water after being submerged for several minutes. In addition to fungi, multiple bacteria were also isolated from sputum and bronchoalveolar lavage of this patient. Patient recovered fully with prolonged treatment with liposomal amphotericin B. Another report also highlighted invasive fungal infection with pulmonary involvement due to Rhizomucor pusillus and Aspergillus niger in an immunocompetent patient. This patient had a history of trauma and was successfully treated by multiple surgical debridement and liposomal amphotericin B [18]. In contrast, in our patient exposure to soil or dust or trauma was not found. An extensive workup for immunodeficiency was not done as his previous history was unremarkable for recurrent infections. His blood glycemic levels remained normal throughout the course of treatment despite being in severe sepsis hence $\mathrm{HbA}_{1 \mathrm{C}}$ levels were never obtained.

Diagnosis of invasive mycosis is challenging due to low sensitivity of currently available diagnostic modalities. Culture and histopathology require invasive sampling approaches such as bronchoscopy with BAL, transbronchial lung biopsy, computed tomographic-guided transthoracic needle biopsy and videoassisted thoracoscopic surgery may be required to make a diagnosis. Classic signs and patterns on radiology for invasive mycosis, although are well documented for immunocompromised patient but are less commonly seen and reported in immunocompetent individuals [19]. Antigen detection and PCRbased methods are usually not available in settings with limited resources. All these factors lead to either misdiagnosis or a delayed diagnosis in immunocompetent patients.

In our patient, culture and microscopy of the BAL fluid yielded growth of A. flavus and L. corymbifera. Both of these fungi are a common cause of invasive mycosis and have been reported to cause illness in immune compromised as well as immune-competent patients. Drug of choice to treat invasive pulmonary aspergillosis is voriconazole and for pulmonary mucormycosis is amphotericin B [19]. In our patient, amphotericin B was started after the microscopy and cytology report and voriconazole was subsequently added to this regimen. The patient improved on this therapy with complete resolution of lung infiltrates in two weeks. The deranged renal function was likely due to acute renal injury secondary to sepsis which led to the presentation similar to pulmonary-renal syndrome. The use of corticosteroids in this case was justified due to high suspicion of life-threatening vasculitis which later turned out to be a fungal infection. To our knowledge, pulmonary coinfection with A. flavus and L. corymbifera in an immunocompetent patient presenting as a pulmonary-renal syndrome has not been reported previously. Clinicians should be aware of such cases for prompt appropriate diagnosis and management.

\section{References}

1. Pfaller MA, Pappas PG, Wingard JR. Invasive fungal pathogens: current epidemiological trends. Clin Infect Dis. 2006;43:S3-14.

2. Blin N, Morineau N, Gaillard F, Morin O, N1 Milpied, Harousseau JL, et al. Disseminated mucormycosis associated with invasive pulmonary aspergillosis in a patient treated for post-transplant high-grade non-Hodgkin's lymphoma. Leuk Lymphoma. 2004;45:2161-3.

3. Gupta V, Rajagopalan N, Patil M, Shivaprasad C. Aspergillus and mucormycosis presenting with normal chest $\mathrm{X}$-ray in an immunocompromised host. BMJ Case Rep. 2014; doi:10.1136/bcr-2014-204022.

4. Marukutira T, Huprikar S, Azie N, Quan SP, Meier-Kriesche HU, Horn DL. Clinical characteristics and outcomes in 303 HIV-infected patients with invasive fungal infections: data from the Prospective Antifungal Therapy Alliance registry, a multicenter, observational study. HIV AIDS. 2014;6:39-47.

5. Mahadevaiah AH, Rajagopalan N, Patil M, Shivaprasad C. Coinfection of pulmonary mucormycosis and aspergillosis presenting as bilateral vocal cord palsy. BMJ Case Rep. 2013; doi:10.1136/bcr-2013-009615.

6. Webb BJ, Blair JE, Kusne S, Scott RL, Steidley DE, Arabia FA, Vikram HR. Concurrent pulmonary Aspergillus fumigatus and mucor infection in a cardiac transplant recipient: a case report. Transpl Proc. 2013;45:792-7.

7. Ozhak-Baysan B, Alastruey-Izquierdo A, Saba R, Ogunc D, Ongut G, Timuragaoglu A, et al. Aspergillus alliaceus and Aspergillus flavus co-infection in an acute myeloid leukemia patient. Med Mycol. 2010;48:995-9.

8. Washington WC, Koneman EW. Color atlas and textbook of diagnostic microbiology. Philadelphia: Lippincott Williams \& Wilkins; 2006.

9. Campbell CK, Johnson EM, Warnock DW. Identification of yeasts, in identification of pathogenic fungi. 2nd ed. Oxford: Wiley-Blackwell; 2013. 
10. Cornet M, Mallat H, Somme D, Guérot E, Kac G, Mainardi $\mathrm{JL}$, et al. Fulminant invasive pulmonary aspergillosis in immunocompetent patients; a two-case report. Clin Microbiol Infect. 2003;9:1224-7.

11. Arendrup MC, O'Driscoll BR, Petersen E, Denning DW. Acute pulmonary aspergillosis in immunocompetent subjects after exposure to bark chippings. Scand J Infect Dis. 2006;38:945-9.

12. Butler L, Brockley T, Denning D, Richardson M, Chisholm $\mathrm{R}$, Sinha S, et al. Acute Aspergillus pneumonia associated with mouldy tree bark-chippings, complicated by antiglomerular basement membrane disease causing permanent renal failure. Med Mycol Case Rep. 2013;2:125-7.

13. Russell K, Broadbridge C, Murray S, Waghorn D, Mahoney A. Gardening can seriously damage your health. Lancet. 2008;371:2056.

14. Zhang L, Tian X, Wang P, Zhang H, Feng R. Recurrent pulmonary mucormycosis after lobectomy in a non smoking patient without predisposing risk factors. Braz J Infect Dis. 2012;16:590-3.
15. Sridhar V, Rajagopalan N, Shivaprasad C, Patil M, Varghese J. Acute community acquired Aspergillus pneumonia in a presumed immunocompetent host. BMJ Case Rep. 2012; doi:10.1136/bcr.09.2011.4866.

16. Kimura M, Udagawa S, Makimura K, Satoh K, Toyazaki N, Ito $\mathrm{H}$. Isolation and identification of Rhizomucor pusillus from pleural zygomycosis in an immunocompetent patient. Med Mycol. 2009;47:869-73.

17. Van Dam AP, Pruijm MT, Harinck BI, Gelinck LB, Kuijper EJ. Pneumonia involving Aspergillus and Rhizopus spp. after a near-drowning incident with subsequent Nocardia cyriacigeorgici and N. farcinica coinfection as a late complication. Eur J Clin Microbiol Infect Dis. 2005;24:61-4.

18. Pozo-Laderas JC, Pontes-Moreno A, Robles-Arista JC, Bautista-Rodriguez MD, Candau-Alvarez A, Caro-Cuenca MT, et al. Mixed invasive fungal infection due to Rhizomucor pusillus and Aspergillus niger in an immunocompetent patient. Rev Iberoam Micol. 2013;32(1):46-50.

19. Smith JA, Kauffman CA. Pulmonary fungal infections. Respirology. 2012;17:913-26. 